\title{
Peroxiredoxin 3 is a novel marker for cell proliferation in cervical cancer
}

\author{
JING-XIA HU ${ }^{1}$, QUN GAO ${ }^{2}$ and LIANQIN $\mathrm{LI}^{2}$ \\ Departments of ${ }^{1}$ Pathology and ${ }^{2}$ Obstetrics and Gynecology, Tsinghua University Second Hospital, \\ Beijing 100049, P.R. China
}

Received October 2, 2012; Accepted November 16, 2012

DOI: $10.3892 / b r .2012 .43$

\begin{abstract}
Although peroxiredoxin 3 (Prx3) has been reported to be involved in cervical cancer (CC) carcinogenesis, the significance of Prx3 in CC progression remains unclear. The present study was conducted to investigate the expression features of Prx3 to better understand the mechanism of tumor growth and invasion. Sixty-eight patients with invasive squamous cervical cancer were included in the present study. The status of human papillomavirus (HPV) infection was detected by hybridization and quantitative real-time polymerase chain reaction (qRT-PCR). Immunohistochemistry was performed on paraffin-embedded sections using monoclonal antibodies against Prx3 and Ki67. All samples were positive for high-risk HPV, among which fifty-six samples were positive for HPV16, seven for HPV18 and five for HPV33. The expression of HPV16 E6/E7 was significantly higher in cancer areas compared to the adjacent normal epithelial tissuses. The positive cells for Prx3 and Ki67 were significantly higher in cancer cells compared to normal epitheliums and the staining pattern of Prx3 was consistent with that of Ki67 (Pearson's correlation coefficient was $0.801, \mathrm{P}=0.000$ ). The upregulation of $\operatorname{Prx} 3$ might be a protective response to oxidative stress in the cancer microenvironment. The expression consistency of Prx3 and Ki67 suggests Prx3 to be a potential marker for cell proliferation of CC.
\end{abstract}

\section{Introduction}

The development of cervical cancer (CC) has been causally linked to oncogenes E6 and E7 of high-risk human papillomaviruses (HPV). The HPV E6 and E7 oncogenes integrate into the genome of infected cervical cells and inactivate the tumor-suppressor proteins p53 and retinoblastoma, which

Correspondence to: Dr Lianqin Li, Department of Obstetrics and Gynecology, Tsinghua University Second Hospital, 5 Shijingshan Road, Shijingshan, Beijing 100049, P.R. China

E-mail: 1ilq2005@126.com

Key words: peroxiredoxin, cervical cancer, reactive oxygen species, proliferation results in epithelial immortalization and ultimate malignant transformation through a multistage process (1). Due to active and indefinite growth of cancer cells, reactive oxygen species (ROS) production from the mitochondria is accordingly increased (2). Excessive ROS is likely to induce oxidative stress and subsequent cell apoptosis through mitochondria or/ and direct injury to protein, DNA and lipid (3-5). In fact, the chemo- or radiotherapy for cancers is through ROS increase and apoptotic induction of cancer cells $(6,7)$.

Organisms have developed multiple antioxidant systems to protect against oxidative damage, among which peroxiredoxin 3 (Prx3) plays an important role in regulating cellular ROS level and apoptosis (8). As a mitochondrial scavenger of ROS, Prx3 has been demonstrated to play an antioxidant role under cellular oxidative conditions $(9,10)$ and the involvement of Prx3 in CC carcinogenesis was previously reported (11). Nevertheless, the significance of Prx3 in CC development and/or progression has not been clear. The present study was conducted to investigate the characteristics of Prx3 expression, and thereby contributing to the understanding of the mechanism of tumor growth and invasion.

\section{Materials and methods}

Patients and samples. After the approval of the Institutional Review Boards of Tsinghua University Second Hospital (Beijing, China), we collected invasive squamous cervical cancer (ISCC) samples from patients between August, 2008 and July, 2010. The samples were collected after obtaining informed consent from the patients. The samples were processed into formalin-fixed and paraffin-embedded tissue blocks. At the same time, adjacent normal epithelial tissues were used as the controls.

Examination of HPV infection. HPV-infection status of the samples was examined using HPV gene array test kit (Hybribio Limited, Hong Kong, China). Briefly, DNA was extracted from cervical tissues and amplified by polymerase chain reaction (PCR). The PCR products were then hybridized on HPV genoarray membrane that contained probes corresponding to $21 \mathrm{HPV}$ types. Biotin was used as the positive control and distilled water as the negative control. The membrane was visualized through NBT/BCIP to determine the status of HPV infection. 


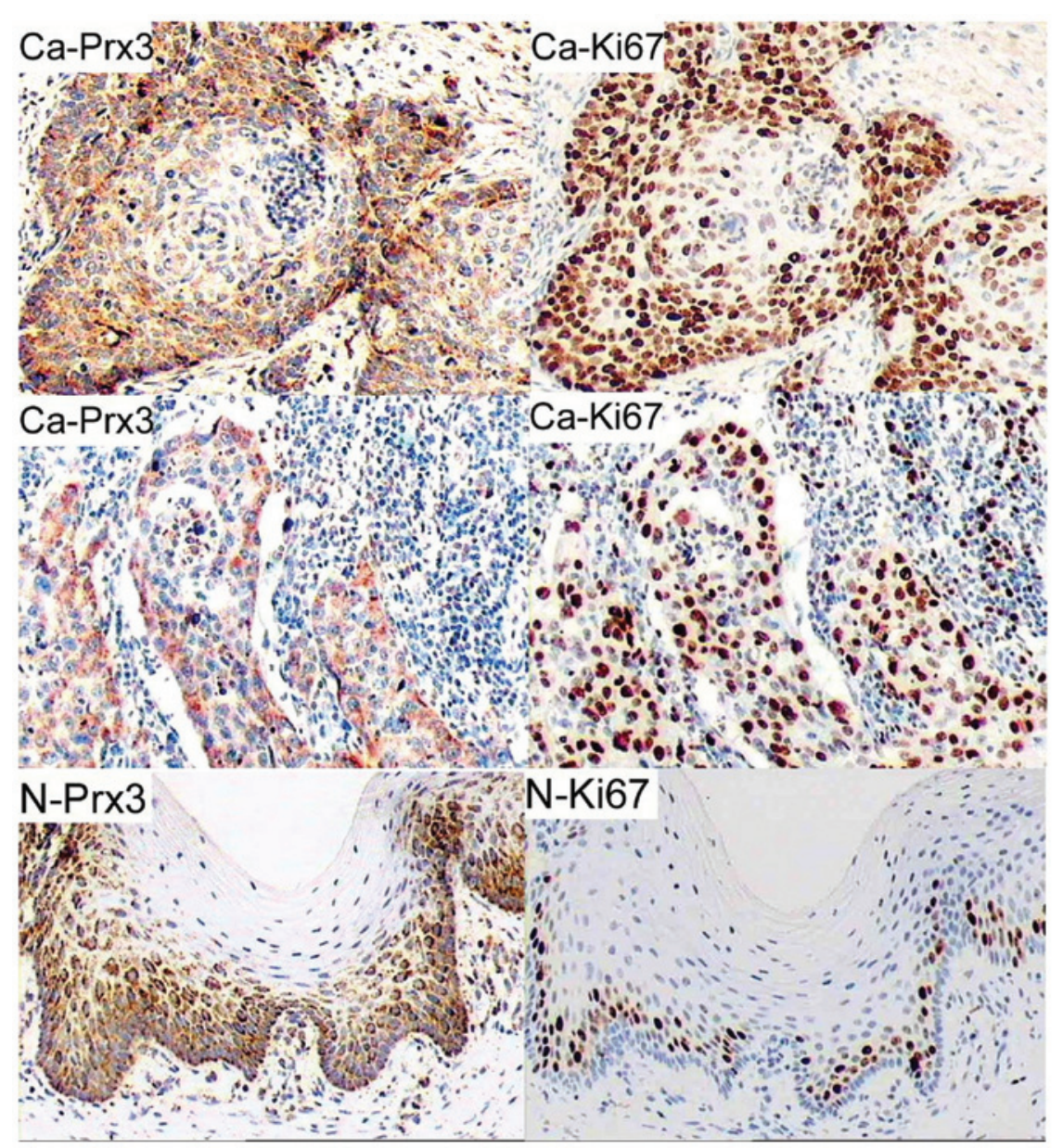

Figure 1. Representative immunohistochemical staining for peroxiredoxin $3(\operatorname{Prx} 3)$ and Ki67 in sections of cervical cancer and adjacent normal epithelium. In cancerous tissues, the positive cells were observed in the cancer nests and surrounding tissues (upper, Ca-Prx3 and Ca-Ki67) or in invaded surrounding areas (middle, Ca-Prx3 and Ca-Ki67). The immunoreactivity was mainly localized in the basal layer of normal epithelium (N-Prx3 and N-Ki67). Original magnification, $\mathrm{x} 200$.

To detect HPV16 mRNA expression in CCs, we performed quantitative real-time PCR (qRT-PCR) using SYBR $^{\circledR}$-GreenER ${ }^{\mathrm{TM}}$ Two-Step qRT-PCR kits (Invitrogen, Carlsbad, CA, USA). Total RNA was extracted from paraffin-embedded tissue sections. After cDNA synthesis, the qRT-PCR program and the calculation for HPV16 expression were performed as previously described (10). The primer sequences for HPV16 E6/E7 were: forward, 5'-GTTACTGCG ACGTGAGGTATATG-3'; reverse, 5'-CATTTATCACATAC AGCATATGGATTC-3'. $\beta$-actin (forward, 5'-ACGTTGACAT CCGAAAGACC-3'; reverse, 5'-CCACCGATCCACACAGA GTA-3') was used as the internal control.

Immunohistochemistry. To detect Prx3 protein in cervical tissues, we performed immunohistochemistry as previously described with a minor modification (9). Consecutive slides were incubated using mouse monoclonal antibodies against human Prx3 and Ki67 respectively (1:1000 dilution for Prx3 and 1:150 dilution for Ki67; Santa Cruz Biotechnology, Inc., Santa Cruz, CA, USA). Immunohistochemical staining scores were evaluated as the percentage of positive cells and counted by two independent persons not aware of the patient information.

\section{Results}

In the present study, we included 68 patients with ISCC (age, $45.96 \pm 9.89$ years). Thirty patients had FIGO stage I and 38 had stage II, of which 12 were cancer-positive in the lymph nodes. The patients did not receive chemo- or radiotherapy prior to the operation.

Expression of high-risk HPV is higher in cancer cells compared to the adjacent normal epitheliums. As determined by the HPV genoarray test kit, all samples were infected with high-risk HPV. Among these, fifty-six samples were positive for HPV16, seven for HPV18 and five for HPV33. In the fifty-six HPV16-positive samples, the expression of HPV16 E6/E7 mRNA relative to $\beta$-actin was significantly higher in cancer samples compared to the adjacent normal epitheliums ( $8996 \pm 409$ vs. $198 \pm 56, \mathrm{P}=0.000$ by analysis of variance).

Expression of Prx 3 and HPV16 is positively correlated in CC samples. As shown in Fig. 1, we observed strong cytoplasmic staining for Prx 3 in the cancer nests and the surrounding cells or in invasive cancerous cells, while Prx3-positive cells in the adjacent epithelium were located 
mainly in the basal layer. The number of positive cells for Prx 3 in cancerous areas was significantly higher compared to that in non-cancerous areas $(65.00 \pm 21.08$ vs. $41.04 \pm 13.09 \%$, $\mathrm{P}=0.000$ by Student's t-test). Of the clinical and pathological characteristics, cell differentiation (grade) was significantly associated with Prx3 expression (Pearson's correlation coefficient was $-0.648, \mathrm{P}=0.000$ ). In addition, the positive cells for Prx3 in CCs were correlated with the expression of HPV16 E6/E7 (Pearson's correlation coefficient was 0.726, $\mathrm{P}=0.000$ ).

Expression of Prx3 and Ki67 is positively correlated in CC samples. Since Prx 3 was predominantly expressed in cells with active proliferation, immunohistochemistry was performed using mouse monoclonal antibody against human Ki67. As shown in Fig. 1, the number of positive cells for Ki67 was significantly higher in cancerous cervices compared to adjacent epitheliums $(70.89 \pm 21.49$ vs. $17.12 \pm 10.28 \%, \mathrm{P}=0.000$ by Student's t-test). Notably, the staining pattern of Prx3 was consistent with that of Ki67 (Fig. 1; Pearson's correlation coefficient was $0.801, \mathrm{P}=0.000$ ). In cancerous sections, Ki67-positive cells were located in the cancer nests and the surrounding areas or in invaded cancerous tissues, while Ki67-positive cells in the adjacent normal epitheliums were located mainly in the basal layer.

\section{Discussion}

In the present study, we demonstrated increased expression of Prx3 in CC and provided evidence that Prx3 was upregulated in the cells with active proliferation. The pattern of Prx3 expression in CC was consistent with that of Ki67, indicating that Prx3 is a potential marker for cell proliferation.

In their study, Riethdorf et al (12) demonstrated that high-risk HPV oncogenes activated telomerase and played a critical role in human carcinogenesis. High-risk HPV genomes were reported to preferentially integrate near the c-myc gene (13). The latter cooperated with HPV E6/E7 to promote the malignant transformation of cervical epitheliums (14). As a target gene of c-myc, Prx3 played an important role in mitochondrial homeostasis maintenance and neoplastic transformation (15). Our findings showed that the HPV16 E6/E7 expression was increased and was positively associated with the expression of Prx 3 in CC. The interaction between HPV, c-myc and Prx 3 in CC development or/and progression requires additional investigation.

As an established marker for cell proliferation, Ki67 has been suggested to be a predictor for high-risk HPV infection and an independent prognostic parameter for recurrence-free survival of CC $(16,17)$. Our findings showed that the Ki67-positive cells were mainly located in the surrounding areas of cancer nests or in invaded cancerous tissues, implying active growth of the cells in these areas. Since ROS production was increased in the cells with active proliferation (2), the upregulation of Prx3 in the same areas might be a natural response to oxidative stress in the cancer microenvironment. In their previous study, Nonn et al (18) reported that Prx3 played a protective role against drug-induced oxidative stress and subsequent apoptosis of cancer cells, thus it would be useful to investigate the significance of $\operatorname{Prx} 3$ in the prognosis, recurrence and chemo- or radiotherapy-resistance of CC.

\section{References}

1. Scheffner M, Munger K, Byrne JC and Howley PM: The state of the p53 and retinoblastoma genes in human cervical carcinoma cell lines. Proc Natl Acad Sci USA 88: 5523-5527, 1991.

2. Beevi SS, Rasheed MH and Geetha A: Evidence of oxidative and nitrosative stress in patients with cervical squamous cell carcinoma. Clin Chim Acta 375: 119-123, 2007.

3. Cotran RS, Kumar V and Robbins SL: Robbins Pathologic Basis of Disease. 5th edition. W.B. Saunders Company, Philadelphia, 1994.

4. Ding B, Chi SG, Kim SH, et al: Role of p53 in antioxidant defense of HPV-positive cervical carcinoma cells following $\mathrm{H}_{2} \mathrm{O}_{2}$ exposure. J Cell Sci 120: 2284-2294, 2007.

5. Singh M, Sharma H and Singh N: Hydrogen peroxide induces apoptosis in HeLa cells through mitochondrial pathway. Mitochondrion 7: 367-373, 2007.

6. Srinivas P, Gopinath G, Banerji A, Dinakar A and Srinivas G: Plumbagin induces reactive oxygen species, which mediate apoptosis in human cervical cancer cells. Mol Carcinog 40: 201-211, 2004.

7. Alexandre J, Batteux F, Nicco C, et al: Accumulation of hydrogen peroxide is an early and crucial step for paclitaxelinduced cancer cell death both in vitro and in vivo. Int J Cancer 119: 41-48, 2006.

8. Chang TS, Cho CS, Park S, Yu S, Kang SW and Rhee SG: Peroxiredoxin III, a mitochondrion-specific peroxidase, regulates apoptotic signaling by mitochondria. J Biol Chem 279: 41975-41984, 2004.

9. Li L, Shoji W, Takano H, et al: Increased susceptibility of MER5 (peroxiredoxin III) knockout mice to LPS-induced oxidative stress. Biochem Biophys Res Commun 355: 715-721, 2007.

10. Li L, Shoji W, Oshima H, Obinata M, Fukumoto M and Kanno N: Crucial role of peroxiredoxin III in placental antioxidant defense of mice. FEBS Lett 582: 2431-2434, 2008.

11. Kim K, Yu M, Han S, et al: Expression of human peroxiredoxin isoforms in response to cervical carcinogenesis. Oncol Rep 21: 1391-1396, 2009.

12. Riethdorf S, Riethdorf L, Schulz G, et al: Relationship between telomerase activation and HPV 16/18 oncogene expression in squamous intraepithelial lesions and squamous cell carcinomas of the uterine cervix. Int J Gynecol Pathol 20: 177-185, 2001.

13. Couturier J, Sastre-Garau X, Schneider-Maunoury S, Labib A and Orth G: Integration of papillomavirus DNA near myc genes in genital carcinomas and its consequences for proto-oncogene expression. J Virol 65: 4534-4538, 1991.

14. Subramanyam D and Krishna S: c-Myc substitutes for Notch1-CBF1 functions in cooperative transformation with papillomavirus oncogenes. Virology 347: 191-198, 2006.

15. Wonsey DR, Zeller KI, and Dang CV: The c-Myc target gene PRDX3 is required for mitochondrial homeostasis and neoplastic transformation. Proc Natl Acad Sci USA 99: 6649-6654, 2002

16. Mimica M, Tomić S, Kardum G, Hofman ID, Kaliterna V and Pejković L: Ki-67 quantitative evaluation as a marker of cervical intraepithelial neoplasia and human papillomavirus infection. Int J Gynecol Cancer 20: 116-119, 2010.

17. Hanprasertpong J, Tungsinmunkong $\mathrm{K}$, Chichareon $\mathrm{S}$, et al: Correlation of p53 and Ki-67 (MIB-1) expressions with clinicopathological features and prognosis of early stage cervical squamous cell carcinomas. J Obstet Gynaecol Res 36: 572-580, 2010.

18. Nonn L, Berggren M and Powis G: Increased expression of mitochondrial peroxiredoxin-3 (thioredoxin peroxidase-2) protects cancer cells against hypoxia and drug-induced hydrogen peroxide-dependent apoptosis. Mol Cancer Res 1: 682-689, 2003. 